\title{
Implantable devices: the future of blood monitoring?
}
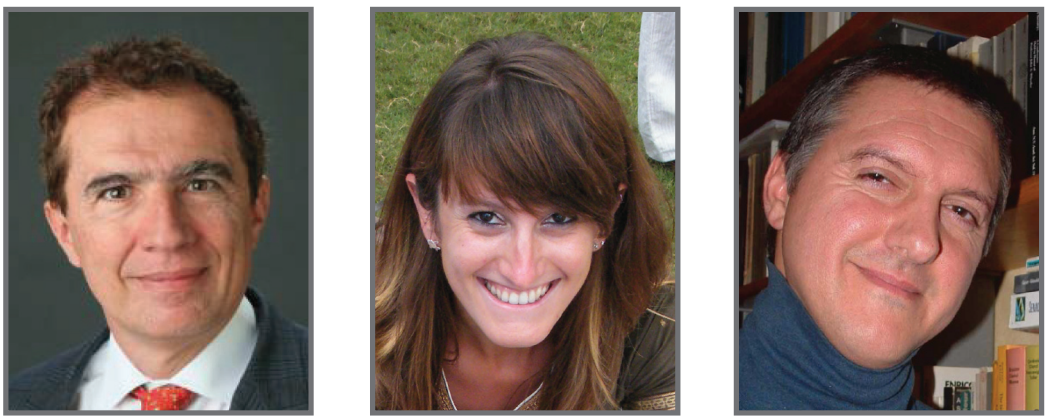

Giovanni De Micheli ${ }^{*}$, Cristina Boero ${ }^{1} \&$ Sandro Carrara ${ }^{1}$

“...implantable devices can be developed for the simultaneous monitoring of a specific group of key metabolites, identified by more complex techniques, such as mass spectroscopy- or nuclear magnetic resonance-based analysis."

Blood analysis is one of the first screening tests used to determine the general status of a person. From such exams it is possible to determine a biochemical analysis of the main metabolites, trace molecular profiles of some organs and evaluate the cellular state of the blood. Among all the important information that can be extracted from the compounds present in the bloodstream, the identification and monitoring of metabolites is a key issue to predict pathological conditions, such as ischemia or stroke, or to keep a chronic disease under control. Indeed, certain metabolites can be up- or down-regulated in the blood due to acute or chronic illnesses. A complete list of human metabolites has been collected in the Human Metabolome Database, which counts around 6500 metabolites gathered from books, journal articles and electronic databases [1]. Metabolomics, which is the comprehensive analysis of low-weight compounds, is a powerful tool to predict and understand several pathologies, such as diabetes, obesity, cardiovascular diseases and even cancer, by accurately describing the profile of biological status [2]. Blood tests are typically carried out on a blood sample extracted from the vein of the patient in the clinic and subsequently analyzed in a laboratory. Mass spectroscopy (MS) and nuclear magnetic resonance (NMR) are very powerful techniques that offer the possibility of metabolomic investigation with amazing precision [3]. However, MS and NMR are expensive and barely portable techniques. Moreover, since blood testing is a 'discrete' analysis, it is not suitable to follow chronic diseases or potentially dangerous conditions continuously and it cannot be used to adjust a pharmacological treatment.

Implantable devices may genuinely represent a valid alternative for continuous monitoring, minimizing the pain and the discomfort of the person. It is always complicated and sometimes dangerous to implant systems that have to be in direct contact with the blood. Thrombosis is the 
"Since there is a strong correlation between metabolite concentration in blood and in the extracellular space, often it is more convenient to locate the implant into the subcutaneous tissues, to avoid coagulation problems and long-term pharmacological therapies." main side effect of such implants, which forces the patient to receive long-term anticoagulation therapies, increasing the risk of bleeding. Since there is a strong correlation between metabolite concentration in blood and in the extracellular space, often it is more convenient to locate the implant into the subcutaneous tissues, to avoid coagulation problems and long-term pharmacological therapies. An outstanding example of partial system implantation is the GlucoDay ${ }^{\circledR}$ (Menarini Diagnostics) to monitor glucose trends over several days $[4,5]$.

In the following section, we describe very recent advancements in the field of fully implantable devices for the remote monitoring of the human metabolism. Indeed, we developed a novel system consisting of an implantable and a wearable device for the continuous and simultaneous monitoring of several metabolites. Then, in the 'Future perspective' we discuss the outstanding implications, brought from our solution, for clinical practice and therapeutic scopes.

\section{Fully implantable devices}

Among all the metabolites listed in the Human Metabolome Database, some of them can be sensed by existing technologies that are suitable to be implanted, such as electrochemical-based biosensors. Current disposable point-of-care devices are used today, but they do not support continuous analysis. Instead, implantable devices can be developed for the simultaneous monitoring of a specific group of key metabolites, identified by more complex techniques, such as MS- or NMR-based analysis. They can definitely play the role of technologies complementary to more general metabolomic techniques, and represent the solution for chronic patients.

For this purpose, we designed and realized a novel system consisting of an implantable and wearable device for the continuous monitoring of human metabolism. The implantable portion of the device consists of a cylinder $2.2 \mathrm{~mm}$ in diameter and $15 \mathrm{~mm}$ in length to be inserted in the interstitial tissues. The device houses several electrochemical biosensors for the simultaneous monitoring of metabolites and transmits data downstream through an information system [6]. The platform is then encapsulated in a flexible outer casing with an opening that hosts a membrane [7]. The membrane allows the metabolites to get in touch with the biosensors. Experiments in mice have shown low levels of toxicity of this implant with the current encapsulation. Glucose, lactate, glutamate and ATP have been already detected in buffer solution as a proof of concept of the device [8-10]. Due to the limited size of the implantable device, batteries cannot be hosted in the tiny implant. The device works in connection with a patch (i.e., the wearable device, located on top of the skin in the body area where the implant is placed). The patch carries out the power transmission, the data receiving and the data retransmission onto longer distances. The patch communicates also via a Bluetooth connection to portable devices, such as smartphone, tablet or an external control station [11]. The doctor, for example, can remotely choose the metabolite to monitor and follow the behavior in realtime, through a dedicated interface on his/her smartphone or tablet. Otherwise, data can be stored in the memory of the phone or laptop and analyzed by the expert later.

\section{Future perspective}

As mentioned, the system described in the previous section was tested for the detection of glucose, lactate, glutamate and ATP. Glucose is certainly the most widely studied metabolite, because it is crucial to the physiology of the human body. For a long time, diabetes has driven the research and development of devices for the monitoring of glucose. However, glycemia is also influenced by numerous other factors independent from diabetes, like fasting due to interventions in the hospital or administration of some drugs (i.e., corticosteroids and vasopressors). In those cases, the continuous monitoring of glucose levels may alert doctors and nursing staff in case the patient is risking hypo- or hyper-glycemic conditions [12]. Healthy cells contain high intracellular ATP concentrations to ensure enough available energy currency. Necrosis due to ischemia or inflammation processes causes cell rupture and massive ATP release into the extracellular matrix. Therefore, ATP monitoring becomes of particular interest in chronic inflammation, such as inflammatory bowel disease and rheumatoid arthritis [13]. The concentration of lactate in extracellular fluids is physiologically low, while it dramatically increases during hypoxia or ischemia and it may create a hostile environment for neighboring cells [14]. Glutamate is the major excitatory neurotransmitter in the brain and is important in synaptic plasticity, axonal 
development and neurodegenerative diseases. Glutamate dysregulation is often associated with multiple psychiatric and cognitive disorders [15]. The aforementioned compounds are single biomarkers for a specific disease. More often, however, it is necessary to monitor a group of metabolites in order to get a more complete overview of the pathology. Besides the straightforward application of an implantable sensor for the monitoring of glycemia in diabetic people, our recent development has demonstrated that it is possible to envisage many other scopes for implantable/wearable devices. Perioperative sensing of glucose, glutamate, glycerol and lactate, for example, may be supportive to prevent cerebral metabolic deterioration during brain surgery [16]. Glucose, lactate, pyruvate and glycerol, however, can be analyzed after liver transplantation, in order to avoid graft failure and retransplantation [17]. Tumor metabolism can also be analyzed by following specific biomarkers. Griffiths et al. have shown that the mutant tumor has a significant decrease in total ATP content with respect to normal tissue [18].

From this perspective, electrochemical biosensors offer numerous advantages to be applied to implantable devices, including system miniaturization and very high versatility. The novelties introduced by this experience have impacts in both the medical and the engineering fields. From the point of view of clinical practice, a platform with multiple detection sites may be implanted in proximity to the graft to monitor the follow-up of the surgery in the early months after transplantation. A similar application can be exploited in the case of tumor resection. Sometimes biomarkers detected in serum may not be distinctive enough to evaluate the success of a resection. Instead, local biomarker levels can often give a better description of the resection follow-up. Indeed, short-term detection of cancer

\section{References}

1 Wishart DS, Knox C, Guo AC et al. HMDB: a knowledgebase for the human metabolome. Nucleic Acids Res. 37(Database Issue), D603-D610 (2009).

2 Bain JR, Stevens RD, Wenner BR et al. Metabolomics applied to diabetes research: moving from information to knowledge. Diabetes 58(11), 2429-2443 (2009).

3 Xiao JF, Varghese RS, Zhou B et al. LC-MS based serum metabolomics for identification of hepatocellular carcinoma biomarkers in

biomarkers in loco would be useful in revealing recurrence of fast growing cells [19]. In the case of inflammation, ATP needs to be detected close to the inflamed site [20], since the molecule has a short half-life and it is quickly converted into adenosine [13].

From a more engineering perspective instead, the multi-sensor platform was conceived in a modular way, which is significantly original for this kind of device. In the case of sensors, and more generally in the case of new implants, the path to commercialization is very long and expensive. Devices for glucose monitoring are the only exception, where a market exists and prospers. Thus, designing sensors for new metabolites may incur high non-recurring engineering costs, if the manufacturing steps are not well planned. However, if sensor microarrays are designed in a modular fashion, similarly to what is done in microelectronics, the nonrecurring engineering costs drop significantly. Our prototype hosts five areas that can be customized to sense different metabolites at a later stage, as well as programmable readout electronics. Therefore, the notion of the common sensing platform helps to reduce development costs, time to human trials and manufacturing costs because of the higher volume of production, eventually resulting in the advance of science, technology and medicine.

Financial \& competing interests disclosure

The authors have no relevant affliations or financial involvement with any organization or entity with a financial interest in or financial conflict with the subject matter or materials discussed in the manuscript. This includes employment, consultancies, honoraria, stock ownership or options, expert testimony, grants or patents received or pending, or royalties.

No writing assistance was utilized in the production of this manuscript.

Egyptian cohort. J. Proteome Res. 11(12), 5914-5923 (2012).

4 Poscia A, Mascini M, Moscone D et al. A microdialysis technique for continuous subcutaneous glucose monitoring in diabetic patients (part 1). Biosens. Bioelectron. 18(7), 891-898 (2003).

5 Varalli M, Marelli G, Maran A et al. A microdialysis technique for continuous subcutaneous glucose monitoring in diabetic patients (part 2). Biosens. Bioelectron. 18(7), 899-905 (2003).
6 Ghoreishizadeh S, Carrara S, De Micheli G. Circuit design for human metabolites biochip. Presented at: IEEE Biomedical Circuits and Systems Conference (BioCAS). San Diego, CA, USA, 10-12 November 2011.

7 Carrara S, Ghoreishizadeh S, Olivo J et al. Fully integrated biochip platforms for advanced healthcare. Sensors 12(8), 11013-11060 (2012).

8 Cavallini A, Baj-Rossi C, Ghoreishizadeh S, De Micheli G, Carrara S. Design, fabrication, and test of a sensor array for perspective 
biosensing in chronic pathologies. Presented at: IEEE Biomedical Circuits and Systems Conference (BioCAS). Hsinchu, Taiwan, 28-30 November 2012.

9 Cavallini A, De Micheli G, Carrara S. Comparison of three methods of biocompatible multi-walled carbon nanotubes confinement for the development of implantable amperometric adenosine-5'triphosphate biosensors. Sensor Lett. 9(5), 1-6 (2011).

10 De Micheli G, Boero C, Baj-Rossi C, Taurino I, Carrara S. Integrated biosensors for personalized medicine. Presented at: 49th Design Automation Conference (DAC). San Francisco, CA, USA, 3-7 June 2012.

11 Olivo J, Carrara S, De Micheli G. Iron IC patch: a wearable device for the remote powering and connectivity of implantable systems. Proceedings of the IEEE International Instrumentation and Measurement Technology Conference (I2MTC). 286-289 (2012).
12 Mader JK, Feichtner F, Bock G et al. Microdialysis - a versatile technology to perform metabolic monitoring in diabetes and critically ill patients. Diabetes Res. Clin. Pract. 97(1), 112-118 (2012).

13 Eltzschig HK, Sitkovsky MV, Robson SC. Purinergic signaling during inflammation. N. Engl. J. Med. 367(24), 2322-2333 (2012).

14 Li X, Zhao L, Chen Z et al. Continuous electrochemical monitoring of extracellular lactate production from neonatal rat cardiomyocytes following myocardial hypoxia. Anal. Chem. 84(12), 5285-5291 (2012).

15 Qin S, van der Zeyden M, Oldenziel WH, Cremers T, Westerink B. Microsensors for in vivo measurement of glutamate in brain tissue. Sensors 8(11), 6860-6884 (2008).

16 Bossers SM, de Boer RDH, Boer C, Peerdeman SM. The diagnostic accuracy of brain microdialysis during surgery: a qualitative systematic review. Acta Neurochir. 155(2), 345-353 (2013).
17 Ungerstedt J, Nowak G, Ungerstedt U, Ericzon BG. Microdialysis monitoring of porcine liver metabolism during warm ischemia with arterial and portal clamping. Liver Transpl. 15(3), 280-286 (2009).

18 Griffiths JR, McSheehy PMJ, Robinson SP et al. Metabolic changes detected by in vivo magnetic resonance studies of HEPA-1 wildtype tumors and tumors deficient in hypoxiainducible factor-1 (HIF-1): evidence of an anabolic role for the HIF-1 pathway. Cancer Res. 62(3), 688-695 (2002).

19 Daniel KD, Kim GY, Vassiliou CC et al. Implantable diagnostic device for cancer monitoring. Biosens. Bioelectron. 24(11), 3252-3257 (2009).

20 Schenk U, Frascoli M, Proietti M et al. ATP inhibits the generation and function of regulatory $\mathrm{T}$ cells through the activation of purinergic P2X receptors. Sci. Signal. 4(162), ra12 (2011) 